\title{
Twisted, Z-Shaped Perylene Bisimide
}

Faysal Ilhan, ${ }^{\dagger \neq}$ Daniel S. Tyson, ${ }^{\dagger,}$, Daniel J. Stasko ${ }^{\xi}$, Kristin Kirschbaum ${ }^{\zeta}$, and Michael A. Meador, ${ }^{* \dagger}$

\author{
${ }^{\dagger}$ Polymers Branch, Materials Division \\ NASA Glenn Research Center, \\ 21000 Brookpark Road, \\ Cleveland, Ohio 44135, \\ And \\ Ohio Aerospace Institute, \\ 22800 Cedar Point Road, \\ Cleveland, Ohio 44142 \\ And \\ ${ }^{\xi}$ The University of Southern Maine \\ Lewis-Auburn College \\ 51 Westminster Street \\ Lewiston, ME 04240 \\ And \\ ${ }^{\zeta}$ Department of Chemistry \\ University of Toledo \\ Toledo, $\mathrm{OH} 44306$
}


Supporting Information

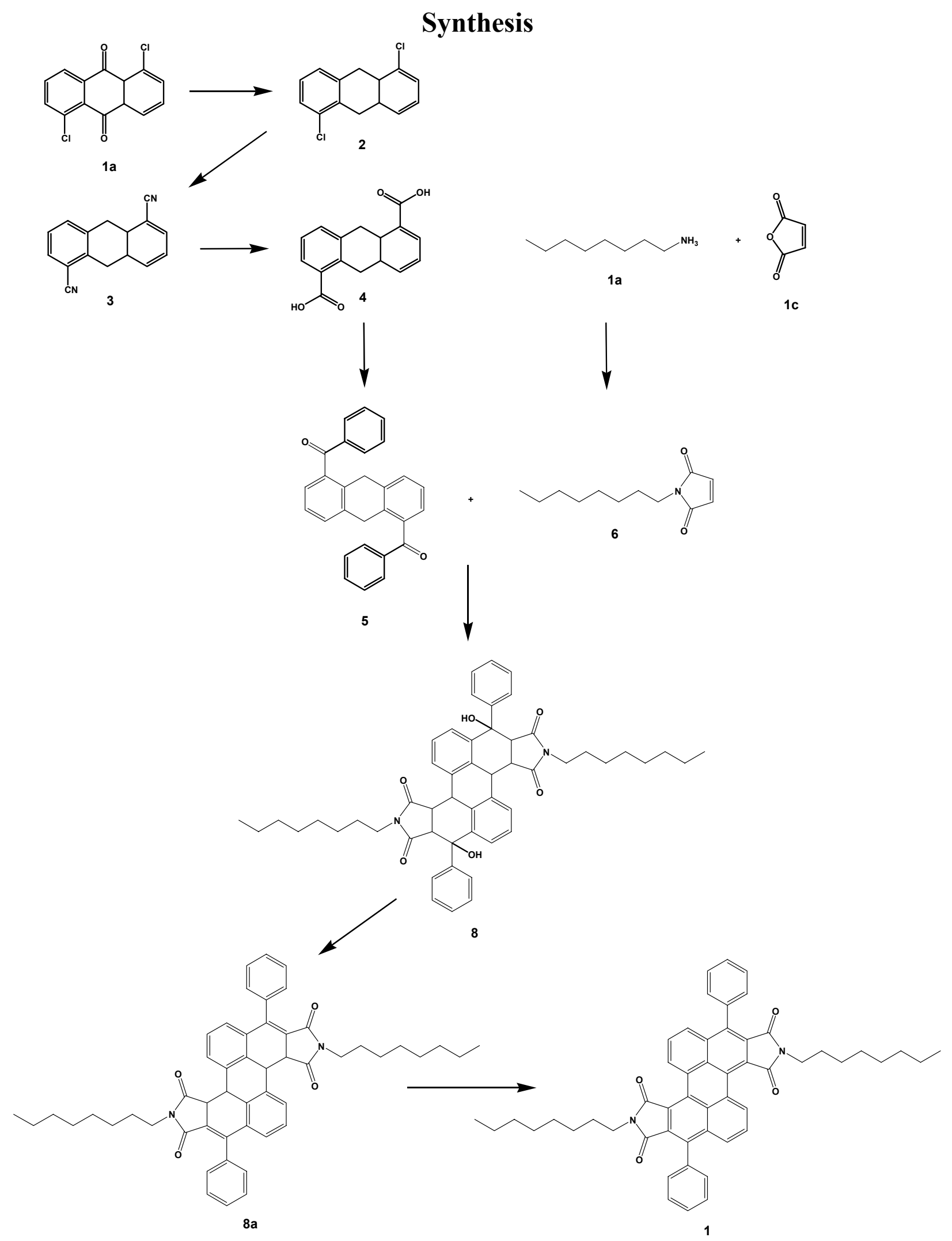

Supporting Scheme 1. Total synthesis of Z-Shaped perylene bisimide (1). 
Materials. All reagents were purchased from Aldrich and used as received. All ${ }^{1} \mathrm{H}$ NMR and ${ }^{13} \mathrm{C}$ NMR spectra $\left(200 \mathrm{MHz}\right.$ or $400 \mathrm{MHz}$ ) were recorded using $\mathrm{CDCl}_{3}$ or $\mathrm{d}_{6}$-DMSO as solvent. Flash column chromatography was performed on silica with an Argonaut Flash Master II system equipped with a UV-Vis absorption detector. Elemental analysis was obtained by Atlantic Microlabs, Inc. (Norcross, GA).

\section{1,5-dichloro-9,10-dihydro anthracene (2):}

A mixture of 1,5-dichloro anthraquinone (1a, $10 \mathrm{~g}, 36 \mathrm{mmol})$, red phosphorous (4.5 $\mathrm{g}, 145$ $\mathrm{mmol})$ and hydroiodic acid $(47+\%, 70 \mathrm{~mL})$ in $470 \mathrm{~mL}$ of glacial acetic acid was refluxed under $\mathrm{N}_{2}$ for 4 days. The resulting mixture was cooled to room temperature and poured into $1 \mathrm{~L}$ of water to precipitate a violet colored solid. The solid was collected by filtration, dissolved in 800 $\mathrm{mL}$ of $\mathrm{CH}_{2} \mathrm{Cl}_{2}$, and filtered again. The filtrate was washed once with $100 \mathrm{~mL}$ of $\mathrm{H}_{2} \mathrm{O}$, dried over $\mathrm{Na}_{2} \mathrm{SO}_{4}$ and concentrated under vacuum to yield the yellow colored, crude solid product. Methanol (100 mL) was added to this solid product and filtered again to collect white needles of the desired product $(7.2 \mathrm{~g}, 80 \%)$. ${ }^{1} \mathrm{H} \mathrm{NMR}\left(300 \mathrm{MHz}, \mathrm{CDCl}_{3}\right): \delta 4.10(\mathrm{~s}, 4 \mathrm{H}), 7.12-7.25(\mathrm{~m}, 6$ H). $\left.{ }^{13} \mathrm{C} \mathrm{NMR} \mathrm{(300} \mathrm{MHz,} \mathrm{CDCl}_{3}\right): \delta 32.93,126.50,127.13,127.20,133.15,137.16$.

\section{1,5-dicyano-9,10-dihydro anthracene (3):}

A mixture of 1,5-dichloro-9,10-dihydro anthracene $(2,6.00 \mathrm{~g}, 24.1 \mathrm{mmol})$, tris(dibenzylideneacetone)dipalladium(0) (8 mol\%, $1.776 \mathrm{~g}), 1,1^{\prime}$-bis(diphenylphosphino)ferrocene (16 mol\%, $2.148 \mathrm{~g})$, zinc (48 mol\%, $0.768 \mathrm{~g})$, and zinc cyanide $(6.84 \mathrm{~g}, 58.3 \mathrm{mmol})$ in $120 \mathrm{~mL}$ of dry dimethyl acetamide $(120 \mathrm{~mL})$ was stirred under $\mathrm{N}_{2}$ at $150{ }^{\circ} \mathrm{C}$ for 2 days. The resulting mixture was cooled to room temperature and poured into $700 \mathrm{~mL}$ of water to precipitate a brown colored solid. The solid was collected by filtration, dissolved in $900 \mathrm{~mL}$ of $\mathrm{CH}_{2} \mathrm{Cl}_{2}$ and filtered again to collect the filtrate. The filtrate was then washed once with $100 \mathrm{~mL}$ of $\mathrm{H}_{2} \mathrm{O}$, dried over $\mathrm{Na}_{2} \mathrm{SO}_{4}$, and concentrated under vacuum to yield a brown colored, crude solid product. Methanol (300 $\mathrm{mL}$ ) was added to this solid product and filtered again to collect a dark cream colored product. The solid was dissolved in $300 \mathrm{~mL}$ of $\mathrm{CH}_{2} \mathrm{Cl}_{2}$ and filtered through silica. The filtrate was treated with charcoal, filtered, and finally evaporated to dryness to yield pale yellow colored needles of the desired product $(3.4 \mathrm{~g}, 61 \%)$. ${ }^{1} \mathrm{H} \mathrm{NMR}\left(300 \mathrm{MHz}, \mathrm{CDCl}_{3}\right): \delta 4.23(\mathrm{~s}, 4 \mathrm{H}), 7.34-7.62(\mathrm{~m}, 6$ 
H). ${ }^{13} \mathrm{C}$ NMR $\left(300 \mathrm{MHz}, \mathrm{CDCl}_{3}\right): \delta 33.88,111.58,117.60,127.23,131.01,132.36,135.92$, 138.52 .

\section{9,10-dihydro anthracene-1,5-dicarboxylic acid (4):}

A mixture of 1,5-dicyano-9,10-dihydro anthracene (3, $9 \mathrm{~g}, 36.9 \mathrm{mmol})$ and $\mathrm{KOH}(32.2 \mathrm{~g}, 0.57$ mol) in $100 \mathrm{~mL}$ ethanol. The solution was refluxed under nitrogen with stirring for 18 hours. Water $(20 \mathrm{~mL})$ and $\mathrm{HCl}(20 \mathrm{~mL})$ were added successively resulting in an orange-yellow precipitate. The mixture was added to $500 \mathrm{~mL}$ of water, filtered, and the solid was collected and dried under vacuum. The resulting green solid product was collected with a quantitative yield (According to NMR studies the product is $90+\%$ pure). ${ }^{1} \mathrm{H}$ NMR (300 $\mathrm{MHz}, \mathrm{d}_{6^{-}}$ DMSO): $\delta 4.34$ (s, 4H), 7.25-7.30 (m, 2 H), 7.51 (d, 2H, J=6 Hz), 7.70 (d, 2H, J=6 Hz), 13.00 (broad s, 2H). ${ }^{13} \mathrm{C}$ NMR (300 MHz, $\left.\mathrm{CDCl}_{3}\right): \delta 33.26,125.78,127.84,129.50,131.01,137.39$, $138.08,168.80$.

\section{1,5-dibenzoyl-9,10-dihydro anthracene (5):}

A solution of 9,10-dihydro anthracene-1,5-dicarboxylic acid (4, $1 \mathrm{~g}, 3.73 \mathrm{mmol})$ in $200 \mu \mathrm{l}$ of dimethyl formamide and $20 \mathrm{~mL}$ of thionyl chloride was refluxed for 3 hours. The resulting solution was evaporated to dryness under vacuum, $60 \mathrm{~mL}$ of benzene added, and warmed to 60 ${ }^{\circ} \mathrm{C}$ to obtain a solution. This solution was slowly added to a $250 \mathrm{~mL}$ round bottom flask containing a mixture of $\mathrm{AlCl}_{3}$ (4 eq.) and $20 \mathrm{~mL}$ of benzene. The resulting mixture was stirred for 18 hours at room temperature and poured into $100 \mathrm{~mL}$ of an ice/water mixture containing 10 $\mathrm{mL}$ of conc. $\mathrm{HCl}$. The organic layer was separated and the aqueous layer was washed twice with $100 \mathrm{~mL}$ portions of benzene. After drying over $\mathrm{Na}_{2} \mathrm{SO}_{4}$, the combined organic extracts were dried under vacuum to yield a brown colored crude product. This was further purified by flash column chromatography to obtain a dark yellow, oily product $(0.39 \mathrm{~g}, 27 \%)$ (According to NMR studies the product is $80+\%$ pure). ${ }^{1} \mathrm{H}$ NMR (300 MHz, $\left.\mathrm{CDCl}_{3}\right): \delta 4.04(\mathrm{~s}, 4 \mathrm{H}), 7.20-7.86(\mathrm{~m}, 16$ $\mathrm{H}), 7.51(\mathrm{~d}, 2 \mathrm{H}, \mathrm{J}=6 \mathrm{~Hz}), 7.70(\mathrm{~d}, 2 \mathrm{H}, \mathrm{J}=6 \mathrm{~Hz}), 13.00$ (broad s, 2H). ${ }^{13} \mathrm{C}$ NMR $(300 \mathrm{MHz}$, $\left.\mathrm{CDCl}_{3}\right): \delta 33.95,125.48,126.98,128.51,128.55,129.94,130.30,133.24,135.74,137.88$, 197.15. 
N-octyl maleimide (6): ${ }^{\mathbf{1}}$ To a suspension of maleic anhydride (1b, $\left.5.4 \mathrm{~g}, 55 \mathrm{mmol}\right)$ in $150 \mathrm{~mL}$ of benzene, a solution of octyl amine (1a, $6.46 \mathrm{~g}, 50 \mathrm{mmol})$ in $100 \mathrm{~mL}$ of benzene was added. The resulting mixture was stirred at $30{ }^{\circ} \mathrm{C}$ for an hour and $\mathrm{ZnBr}_{2}(12.4 \mathrm{~g}, 55 \mathrm{mmol})$ and hexamethyl disilazane $(12.1 \mathrm{~g}, 75 \mathrm{mmol})$ in $30 \mathrm{~mL}$ of benzene were added. The resulting suspension was refluxed for $2 \mathrm{hrs}$. After cooling to room temperature, the reaction mixture was poured into $200 \mathrm{~mL}$ of $0.5 \mathrm{M} \mathrm{HCl}$. The organic layer was separated and the aqueous portion was extracted twice with $150 \mathrm{~mL}$ portions of EtOAc. The combined organic layers were washed with saturated aqueous $\mathrm{NaHCO}_{3}(2 \times 150 \mathrm{~mL})$, brine $(1 \times 150 \mathrm{~mL})$, and dried over $\mathrm{Na}_{2} \mathrm{SO}_{4}$. The solution was dried under vacuum to yield an oily product which solidified gradually to yield the cream colored solid product $(9.2 \mathrm{~g}, 88 \%) .{ }^{1} \mathrm{H}$ NMR $\left(300 \mathrm{MHz}, \mathrm{CDCl}_{3}\right): \delta 0.87(\mathrm{t}, 3 \mathrm{H}, \mathrm{J}=6 \mathrm{~Hz})$, 1.20-1.40 (m, 10H), 1.50-1.70 (m, 2H), 3.51 (t, 2H, J=6 Hz), 6.69 (s, 2H).

\section{N,N'-Bis(octyl)-3,9-dihydroxy-3,9-bis(phenyl)-1,2,3,7,8,9,13,14-octahydro-perylene-1,2,7,8- tetracarboxyl bisimide (8):}

A solution of 1,5-dibenzoyl-9,10-dihydro anthracene $(5,0.39 \mathrm{~g}, 1 \mathrm{mmol})$ and N-octyl maleimide $(6,0.465 \mathrm{~g}, 2.22 \mathrm{mmol})$ in $330 \mathrm{~mL}$ of benzene was vigorously degassed under $\mathrm{N}_{2}$ for 45 minutes in a reactor fitted with a reflux condenser. The $\mathrm{N}_{2}$ flow was reduced and the solution was stirred for 16 hours under irradiation using a $450 \mathrm{~W}$ medium pressure Hg lamp (Hanovia) equipped with a Pyrex filter. The solvent was removed in vacuo and the resulting crude product was triturated with $\mathrm{CH}_{3} \mathrm{OH}$ to yield the desired off-white colored products as a mixture of possible isomers $(0.4$ g, 49\%). ${ }^{1} \mathrm{H}$ NMR $\left(300 \mathrm{MHz}, \mathrm{CDCl}_{3}\right): \delta 0.40-1.30(\mathrm{~m}, 30 \mathrm{H}), 3.08-3.15(\mathrm{~m}, 4 \mathrm{H}), 3.65-3.70(\mathrm{~m}$, $2 \mathrm{H}), 3.90$ (d, 2H, J=7.5Hz), 4.07 (d, 2H, J=9Hz), $5.71(\mathrm{~s}, 2 \mathrm{H}), 7.20-7.50(\mathrm{~m}, 14 \mathrm{H}), 7.80(\mathrm{~d}, 2 \mathrm{H}$, $\mathrm{J}=6 \mathrm{~Hz}) .{ }^{13} \mathrm{C} \mathrm{NMR}\left(300 \mathrm{MHz}, \mathrm{CDCl}_{3}\right): \delta 14.05,22.55,25.83,26.98,28.67,28.93,31.78,35.32$, $38.68,44.35,48.14,123.98,126.82,127.43,127.77,128.19,128.49,128.91,132.12,140.10$, $141.19,175.26,179.80$.

\section{N,N'-Bis(octyl)-3,9-bis(phenyl)-1,7,13,14-tetrahydro-perylene-1,2,7,8-tetracarboxyl}

\section{bisimide (8a):}

A solution of the photoadduct $(\mathbf{8}, 0.5 \mathrm{~g}, 0.62 \mathrm{mmol})$ and catalytic amount of p-toluene sulfonic acid monohydrate ( $p$-TSA) $(0.11 \mathrm{~g}, 0.58 \mathrm{mmol})$ in $100 \mathrm{~mL}$ of toluene was refluxed for 16 hours. The resulting solution was evaporated to dryness under vacuum. Methanol $(40 \mathrm{~mL})$ was added 
to the resulting crude product and the mixture was stirred to dissolve the $p$-TSA. The mixture was filtered to collect the solid product, which was then used in the following aromatization step without any further purification $(0.265 \mathrm{~g}, 55 \%)$. ${ }^{1} \mathrm{H} \mathrm{NMR}\left(300 \mathrm{MHz}, \mathrm{CDCl}_{3}\right): \delta 0.86(\mathrm{t}, 6 \mathrm{H}, \mathrm{J}=6$ Hz), 1.24-1.34 (m, 20 H), 1.60-1.80 (m, 4H), 3.56 (d, 2H, J=12 Hz), 3.63 (t, 4H, J=6 Hz), 4.62 $(\mathrm{d}, 2 \mathrm{H}, \mathrm{J}=12 \mathrm{~Hz}), 7.03(\mathrm{~d}, 2 \mathrm{H}, \mathrm{J}=6 \mathrm{~Hz}), 7.33(\mathrm{t}, 2 \mathrm{H}, \mathrm{J}=7.5 \mathrm{~Hz}), 7.40-7.60(\mathrm{~m}, 12 \mathrm{H}) .{ }^{13} \mathrm{C}$ NMR $\left(300 \mathrm{MHz}, \mathrm{CDCl}_{3}\right): \delta 14.00,22.58,27.01,27.89,29.11,29.14,31.72,38.94,40.62,46.17$, $124.55,126.34,127.85,129.22,129.28,130.34,130.99$, 133.33, 133.77, 134.59, 135.95, 146.42, $166.16,174.31$.

\section{N,N'-Bis(octyl)-3,9-bis(phenyl)-perylene-1,2,7,8-tetracarboxyl bisimide (1):}

A solution of the dehyrdrated precursor (8a, $0.26 \mathrm{~g}, 0.34 \mathrm{mmol})$, sulfur $(0.16 \mathrm{~g}, 5 \mathrm{mmol})$ in $6 \mathrm{~mL}$ of diphenylether was refluxed under $\mathrm{N}_{2}$ for four hours. The solution was cooled to room temperature and $100 \mathrm{~mL}$ of hexanes were added. The resulting solid was collected by filtration. The solid was stirred in $100 \mathrm{~mL}$ of $\mathrm{CH}_{2} \mathrm{Cl}_{2}$ and filtered. The filtrate was concentrated in vacuo and further purified by flash column chromatography to yield the title product as an orange solid (0.17 g, 66\%). ${ }^{1} \mathrm{H}$ NMR (300 MHz, $\left.\mathrm{CDCl}_{3}\right): \delta 0.85$ (t, 6H, J=6 Hz), 1.24-1.39 (m, $\left.20 \mathrm{H}\right), 1.60-$ $1.80(\mathrm{~m}, 4 \mathrm{H}), 3.68(\mathrm{t}, 4 \mathrm{H}, \mathrm{J}=6 \mathrm{~Hz}), 7.40-7.50(\mathrm{~m}, 4 \mathrm{H}), 7.50-7.70(\mathrm{~m}, 6 \mathrm{H}), 7.70-7.80(\mathrm{~m}, 2 \mathrm{H})$, $7.84(\mathrm{~d}, 2 \mathrm{H}, \mathrm{J}=9 \mathrm{~Hz}), 9.01(\mathrm{~d}, 2 \mathrm{H}, \mathrm{J}=9 \mathrm{~Hz}) .{ }^{13} \mathrm{C} \mathrm{NMR}\left(300 \mathrm{MHz}, \mathrm{CDCl}_{3}\right): \delta 14.04,22.61,27.06$, $28.44,29.17,31.77,38.71,123.35,126.45,128.07,128.26,128.33,128.61,129.93,130.35$, 131.90, 132.58, 133.28, 134.78, 134.92, 139.24, 166.53, 167.98. Anal. Calcd for $\mathrm{C}_{52} \mathrm{H}_{50} \mathrm{~N}_{2} \mathrm{O}_{4}$ : C, 81.43; H, 6.57. Found: C, 80.70; H, 6.54.

\section{Photophysical and Electrochemical}

Analytically pure samples of perylene bisimide (1) were used for all experiments. Purification was achieved using flash column chromatography (silica, hexane:ethyl acetate). Optimal separation was accomplished with a gradient profile; 100\% hexane for 5 minutes followed by an increase of $1 \%$ per min of ethyl acetate. Samples were typically dry loaded onto the column by evaporating a concentrated solution onto a minimum amount of silica. 
Unless otherwise stated, all experiments used optically dilute solutions (OD $<0.2)$ at room temperature. Fresh samples were prepared for all measurements by dissolving the appropriate analyte in dichloromethane. Luminescence solutions were prepared under atmospheric conditions and held in anaerobic $1 \mathrm{~cm}^{2}$ quartz cells (Spectracell, FUV) during interrogation. Absorption spectra were measured with a Shimadzu scanning spectrophotometer (UV-3101PC). Emission spectra were obtained with an Aminco-Bowman luminescence spectrometer (Series 2). The excitation was accomplished with a $150 \mathrm{~W}$ Xe lamp optically coupled to a monochromator $( \pm 2 \mathrm{~nm})$. The emission was collected at $90^{\circ}$ and passed through a second monochromator $( \pm 2 \mathrm{~nm})$. Luminescence was measured with a photomultiplier tube (PMT). Radiative quantum yields $\left(\Phi_{\mathrm{r}}\right)$ were measured against fluorescein for which $\Phi_{\mathrm{r}}=0.90$ in $0.01 \mathrm{M} \mathrm{NaOH}$, accurate to $10 \%,{ }^{2}$ and calculated using the following equation ${ }^{2,3}$

$$
\Phi_{u n k}=\Phi_{s t d}\left(\frac{I_{u n k}}{A_{u n k}}\right)\left(\frac{A_{s t d}}{I_{s t d}}\right)\left(\frac{\eta_{u n k}}{\eta_{s t d}}\right)^{2}
$$

where $u n k$ represents the sample, std represents the standard, $\Phi$ is the radiative quantum yield, $I$ is the integrated emission intensity, $A$ is the absorbance at the excitation wavelength, and $\eta$ is the refractive index of the solvent.

Luminescence lifetimes were measured with an IBH time-correlated single photon counting (TCSPC) system equipped with an IBH Model TBX-04 Photon Detection Module. The excitation source for the TCSPC measurements was a pulsed LED (IBH NanoLED, $455 \mathrm{~nm}, 1.3$ ns pulse duration or $403 \mathrm{~nm},<200$ ps pulse duration) with a repetition rate of $1 \mathrm{MHz}$. All data was analyzed by iterative reconvolution of the decay profile (10,000 counts at the peak channel) with the instrument response function using software provided by the instrument manufacturer.

Electrochemical measurements were recorded with an EG\&G Princeton Applied Research Potentiostat/Galvanostat (Model 273A). The working electrode consisted of a platinum disk, 3 $\mathrm{mm}$ in diameter. A platinum mesh served as a counter electrode and a silver wire was used as a quasi reference electrode. All electrodes were polished with $0.05 \mu \mathrm{m}$ alumina prior to measurements. Solutions were prepared with $100 \mathrm{mM}$ TBAP electrolyte and degassed with argon for 20 minutes prior to each measurement. Reported potentials are versus SCE and were determined by adding ferrocene (taking $\mathrm{E}_{\mathrm{Fc} / \mathrm{Fc}^{+}}^{0}=0.424 \mathrm{~V}$ vs SCE in benzene) as an internal potential marker. ${ }^{4}$ 


\section{Crystallography}

Compound 1 was disordered with respect to the phenyl side chain and octyl side chain. The disordered atoms were modeled isotropically. The octyl side chain was modeled such that the last 3 carbons of the chain $(\mathrm{C} 24, \mathrm{C} 25$, and C26) were fixed to half occupancy shared between two positions which closely matches conformational changes in the segment of the octyl chain. Similarly the phenyl ring was split between two possible conformations. The crystal structure has been deposited at the Cambridge Crystallographic Data Centre and allocated the deposition number CCDC 284153.

Table 1. Crystal data and structure refinement for $\mathbf{1 .}$

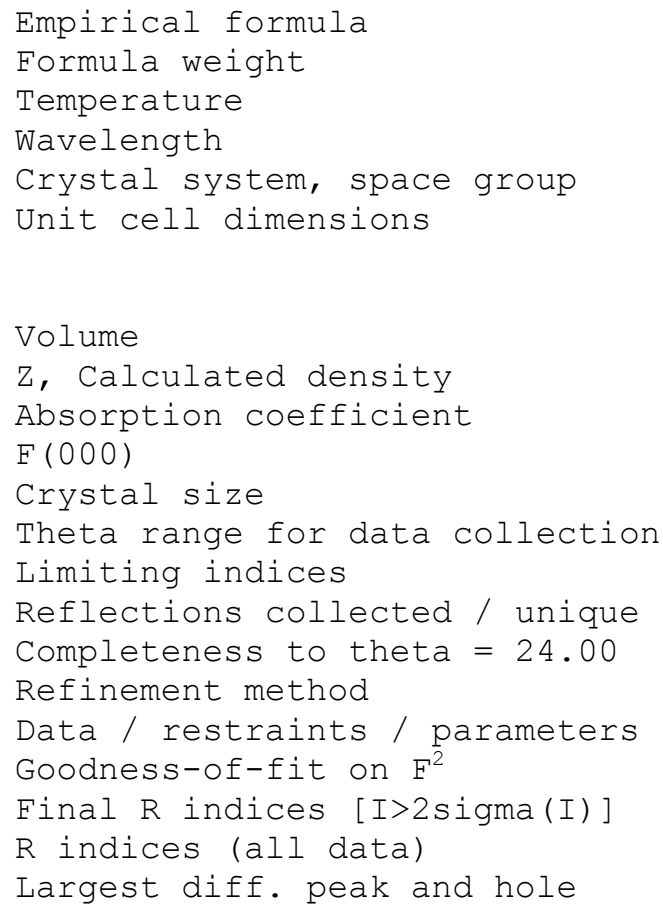

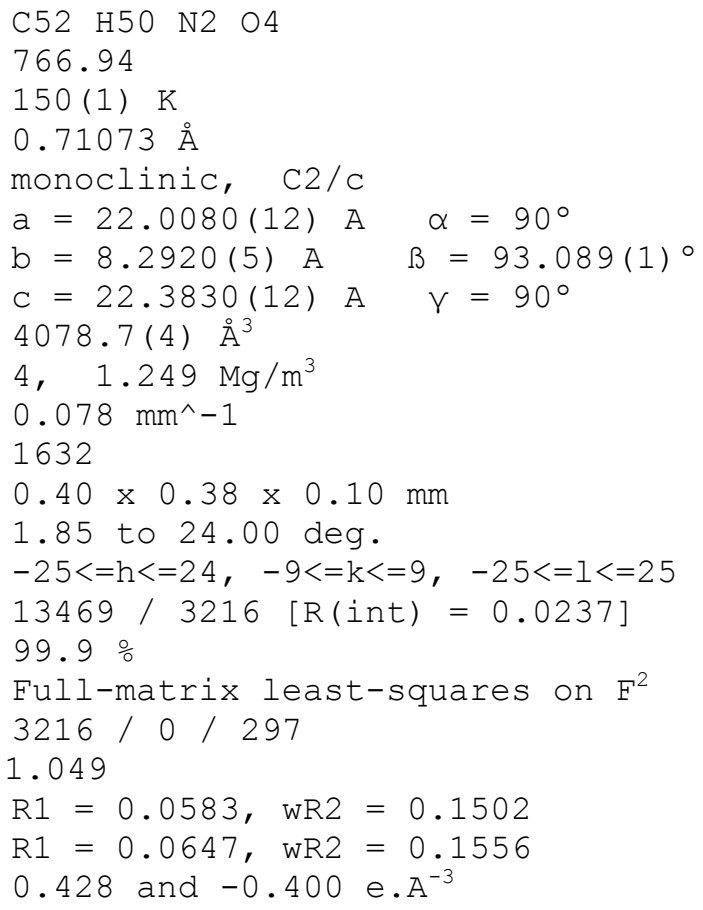


Supporting Figure 1. ORTEP plot of asymmetric unit of $\mathbf{1}$ with labeling.

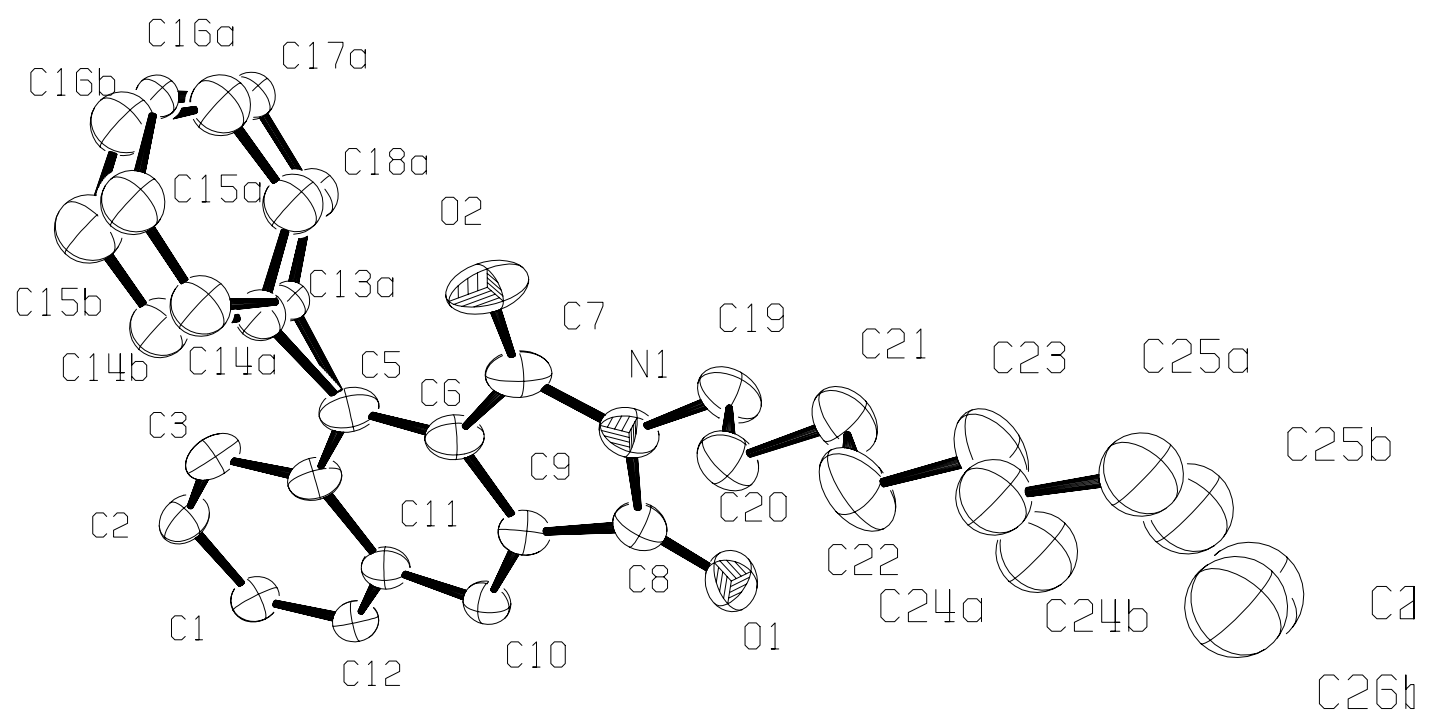

Supporting Figure 2. ORTEP plot of 1, twisted Z-shaped perylene core (2 views, phenyl ring and octyl chain removed for clarity).

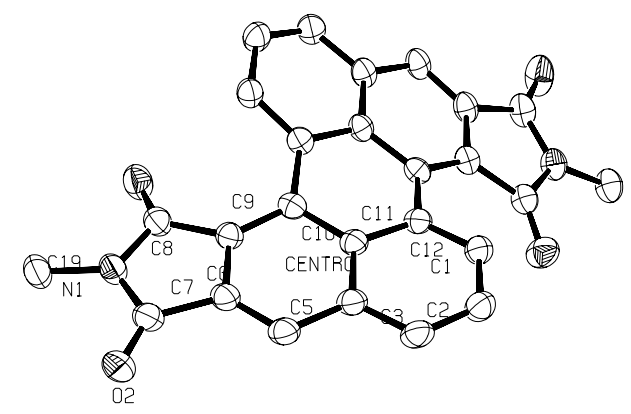

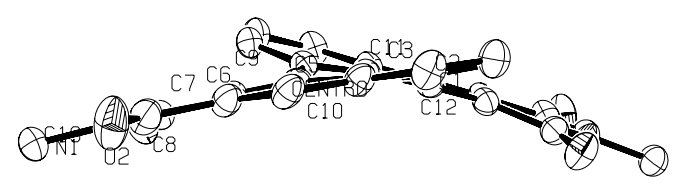


Supporting Information

Supporting Figure 3. Packing diagram of $\mathbf{1}$.

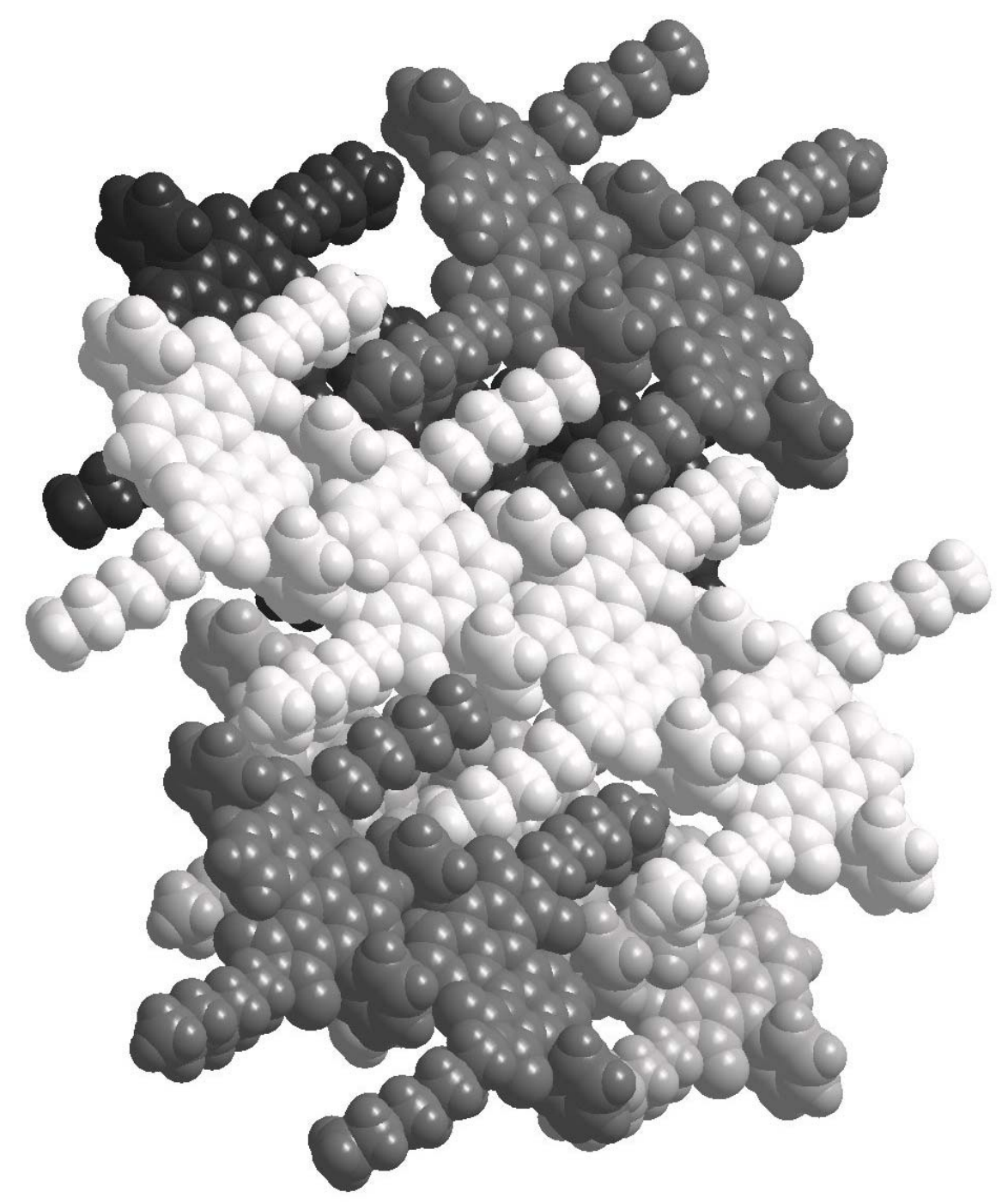


Table 2. Atomic coordinates $\left(\mathrm{x} 10^{4}\right)$ and equivalent isotropic displacement parameters $\left(\AA^{2} \mathrm{x}\right.$ $10^{3}$ ) for 4 . $\mathrm{U}(\mathrm{eq})$ is defined as one third of the trace of the orthogonalized Uij tensor.

\begin{tabular}{|c|c|c|c|c|}
\hline & $x$ & $y$ & z & $\mathrm{U}(\mathrm{eq})$ \\
\hline$O(1)$ & $8272(1)$ & $6521(2)$ & $3227(1)$ & $46(1)$ \\
\hline$O(2)$ & $7421(1)$ & 6095 (3) & $1342(1)$ & $57(1)$ \\
\hline $\mathrm{N}(1)$ & $7723(1)$ & $6343(2)$ & $2332(1)$ & $37(1)$ \\
\hline$C(1)$ & $10686(1)$ & $4540(3)$ & $1498(1)$ & $33(1)$ \\
\hline$C(2)$ & $10407(1)$ & $4393(3)$ & $924(1)$ & $38(1)$ \\
\hline$C(3)$ & $9799(1)$ & $4675(3)$ & $832(1)$ & $38(1)$ \\
\hline$C(4)$ & $9433(1)$ & $5011(3)$ & $1316(1)$ & $33(1)$ \\
\hline$C(5)$ & $8792(1)$ & $5301(3)$ & $1219(1)$ & $35(1)$ \\
\hline$C(6)$ & $8473(1)$ & $5620(3)$ & $1713(1)$ & $34(1)$ \\
\hline$C(7)$ & $7815(1)$ & $6029(3)$ & $1738(1)$ & $40(1)$ \\
\hline$C(8)$ & $8250(1)$ & $6178(3)$ & $2700(1)$ & $35(1)$ \\
\hline C (9) & $8736(1)$ & $5615(2)$ & $2305(1)$ & 31 (1) \\
\hline$C(10)$ & $9348(1)$ & $5253(2)$ & $2420(1)$ & $29(1)$ \\
\hline C (11) & $9710(1)$ & $5078(2)$ & $1908(1)$ & $29(1)$ \\
\hline$C(12)$ & $10356(1)$ & $4954(2)$ & $1984(1)$ & $30(1)$ \\
\hline$C(13 A)$ & $8493(2)$ & $5528(7)$ & $627(2)$ & $22(1)$ \\
\hline$C(14 A)$ & $8205(3)$ & $4150(7)$ & 357 (3) & $48(1)$ \\
\hline$C(15 A)$ & 7940 (3) & $4261(8)$ & $-219(3)$ & $52(2)$ \\
\hline$C(16 A)$ & $7952(2)$ & $5726(7)$ & $-528(2)$ & $25(1)$ \\
\hline$C(17 A)$ & $8193(2)$ & $7094(7)$ & $-238(2)$ & $31(1)$ \\
\hline$C(18 A)$ & $8462(3)$ & $6977(8)$ & $318(3)$ & $37(2)$ \\
\hline$C(13 B)$ & $8509(3)$ & $5079(8)$ & $573(3)$ & $35(2)$ \\
\hline$C(14 B)$ & $8406(3)$ & $3767(7)$ & $274(2)$ & $46(1)$ \\
\hline$C(15 B)$ & $8131(3)$ & $3793(9)$ & $-297(3)$ & $60(2)$ \\
\hline$C(16 B)$ & $7965(3)$ & $5082(9)$ & $-548(3)$ & $53(2)$ \\
\hline$C(17 B)$ & $8104(3)$ & $6513(10)$ & $-301(3)$ & $50(2)$ \\
\hline$C(18 B)$ & $8393(3)$ & $6549(10)$ & $292(3)$ & $50(2)$ \\
\hline C (19) & $7143(1)$ & $6870(3)$ & $2553(1)$ & $42(1)$ \\
\hline$C(20)$ & $6751(1)$ & $5478(3)$ & $2741(1)$ & $42(1)$ \\
\hline$C(21)$ & $6162(1)$ & $6078(3)$ & $2988(1)$ & $47(1)$ \\
\hline C (22) & $5749(1)$ & $4770(3)$ & $3203(2)$ & $64(1)$ \\
\hline C (23) & $5187(1)$ & $5416(4)$ & $3491(2)$ & $64(1)$ \\
\hline$C(24 A)$ & $4676(3)$ & $4097(7)$ & $3562(3)$ & $52(1)$ \\
\hline$C(25 A)$ & $4145(3)$ & $4659(8)$ & $3895(3)$ & $60(2)$ \\
\hline$C(26 A)$ & $4334(4)$ & $4763(11)$ & $4569(4)$ & $97(2)$ \\
\hline$C(24 B)$ & $4864(3)$ & $4254(7)$ & $3865(3)$ & $60(2)$ \\
\hline$C(25 B)$ & $4294(3)$ & $5000(9)$ & $4149(4)$ & $72(2)$ \\
\hline$C(26 B)$ & $3942(3)$ & $3810(9)$ & $4482(3)$ & $84(2)$ \\
\hline
\end{tabular}


Supporting Information

Table 3. Bond lengths $[\AA]$ and angles [deg] for $\mathbf{1}$.

\begin{tabular}{|c|c|}
\hline$O(1)-C(8)$ & $1.211(3)$ \\
\hline$O(2)-C(7)$ & $1.208(3)$ \\
\hline$N(1)-C(7)$ & $1.381(3)$ \\
\hline $\mathrm{N}(1)-\mathrm{C}(8)$ & $1.393(3)$ \\
\hline $\mathrm{N}(1)-\mathrm{C}(19)$ & $1.460(3)$ \\
\hline$C(1)-C(12)$ & $1.383(3)$ \\
\hline$C(1)-C(2)$ & $1.399(3)$ \\
\hline $\mathrm{C}(1)-\mathrm{H}(1)$ & $0.97(2)$ \\
\hline$C(2)-C(3)$ & $1.363(3)$ \\
\hline $\mathrm{C}(2)-\mathrm{H}(2)$ & $0.96(3)$ \\
\hline$C(3)-C(4)$ & $1.413(3)$ \\
\hline $\mathrm{C}(3)-\mathrm{H}(3)$ & $0.94(2)$ \\
\hline$C(4)-C(11)$ & $1.429(3)$ \\
\hline$C(4)-C(5)$ & $1.436(3)$ \\
\hline$C(5)-C(6)$ & $1.367(3)$ \\
\hline$C(5)-C(13 A)$ & $1.459(5)$ \\
\hline$C(5)-C(13 B)$ & $1.555(6)$ \\
\hline$C(6)-C(9)$ & $1.418(3)$ \\
\hline$C(6)-C(7)$ & $1.491(3)$ \\
\hline$C(8)-C(9)$ & $1.499(3)$ \\
\hline$C(9)-C(10)$ & $1.390(3)$ \\
\hline$C(10)-C(11)$ & $1.439(3)$ \\
\hline$C(10)-C(12) \# 1$ & $1.474(3)$ \\
\hline$C(11)-C(12)$ & $1.426(3)$ \\
\hline$C(12)-C(10) \# 1$ & $1.474(3)$ \\
\hline$C(13 A)-C(18 A)$ & $1.386(8)$ \\
\hline$C(13 A)-C(14 A)$ & $1.426(8)$ \\
\hline$C(14 A)-C(15 A)$ & $1.389(8)$ \\
\hline $\mathrm{C}(14 \mathrm{~A})-\mathrm{H}(14 \mathrm{~A})$ & 0.9500 \\
\hline$C(15 A)-C(16 A)$ & $1.398(8)$ \\
\hline$C(15 A)-H(15 A)$ & 0.9500 \\
\hline$C(16 A)-C(17 A)$ & $1.397(7)$ \\
\hline $\mathrm{C}(16 \mathrm{~A})-\mathrm{H}(16 \mathrm{~A})$ & 0.9500 \\
\hline$C(17 A)-C(18 A)$ & $1.352(8)$ \\
\hline$C(17 A)-H(17 A)$ & 0.9500 \\
\hline $\mathrm{C}(18 \mathrm{~A})-\mathrm{H}(18 \mathrm{~A})$ & 0.9500 \\
\hline$C(13 B)-C(14 B)$ & $1.291(9)$ \\
\hline$C(13 B)-C(18 B)$ & $1.389(10)$ \\
\hline$C(14 B)-C(15 B)$ & $1.384(8)$ \\
\hline$C(14 B)-H(14 B)$ & 0.9500 \\
\hline$C(15 B)-C(16 B)$ & $1.252(10)$ \\
\hline$C(15 B)-H(15 B)$ & 0.9500 \\
\hline$C(16 B)-C(17 B)$ & $1.337(10)$ \\
\hline $\mathrm{C}(16 \mathrm{~B})-\mathrm{H}(16 \mathrm{~B})$ & 0.9500 \\
\hline$C(17 B)-C(18 B)$ & $1.439(10)$ \\
\hline $\mathrm{C}(17 \mathrm{~B})-\mathrm{H}(17 \mathrm{~B})$ & 0.9500 \\
\hline $\mathrm{C}(18 \mathrm{~B})-\mathrm{H}(18 \mathrm{~B})$ & 0.9500 \\
\hline$C(19)-C(20)$ & $1.514(3)$ \\
\hline $\mathrm{C}(19)-\mathrm{H}(19 \mathrm{~B})$ & $0.98(3)$ \\
\hline $\mathrm{C}(19)-\mathrm{H}(19 \mathrm{~A})$ & $0.98(3)$ \\
\hline$C(20)-C(21)$ & $1.519(3)$ \\
\hline $\mathrm{C}(20)-\mathrm{H}(20 \mathrm{~B})$ & $1.00(3)$ \\
\hline $\mathrm{C}(20)-\mathrm{H}(20 \mathrm{~A})$ & $1.02(3)$ \\
\hline
\end{tabular}




\begin{tabular}{|c|c|}
\hline$C(21)-C(22)$ & $1.511(4)$ \\
\hline $\mathrm{C}(21)-\mathrm{H}(21 \mathrm{~B})$ & $0.95(3)$ \\
\hline $\mathrm{C}(21)-\mathrm{H}(21 \mathrm{~A})$ & $0.94(3)$ \\
\hline$C(22)-C(23)$ & $1.522(4)$ \\
\hline $\mathrm{C}(22)-\mathrm{H}(22 \mathrm{~A})$ & 0.9900 \\
\hline $\mathrm{C}(22)-\mathrm{H}(22 \mathrm{~B})$ & 0.9900 \\
\hline$C(23)-C(24 B)$ & $1.483(7)$ \\
\hline$C(23)-C(24 A)$ & $1.583(6)$ \\
\hline$C(23)-H(23 B)$ & $1.03(4)$ \\
\hline $\mathrm{C}(23)-\mathrm{H}(23 \mathrm{~A})$ & $0.99(3)$ \\
\hline$C(24 A)-C(25 A)$ & $1.494(8)$ \\
\hline$C(24 A)-H(24 A)$ & 0.9900 \\
\hline$C(24 A)-H(24 B)$ & 0.9900 \\
\hline$C(25 A)-C(26 A)$ & $1.546(10)$ \\
\hline$C(25 A)-H(25 A)$ & 0.9900 \\
\hline$C(25 A)-H(25 B)$ & 0.9900 \\
\hline$C(26 A)-H(26 A)$ & 0.9800 \\
\hline$C(26 A)-H(26 B)$ & 0.9800 \\
\hline$C(26 A)-H(26 C)$ & 0.9800 \\
\hline$C(24 B)-C(25 B)$ & $1.563(9)$ \\
\hline$C(24 B)-H(24 C)$ & 0.9900 \\
\hline$C(24 B)-H(24 D)$ & 0.9900 \\
\hline$C(25 B)-C(26 B)$ & $1.480(10)$ \\
\hline$C(25 B)-H(25 D)$ & 0.9900 \\
\hline$C(25 B)-H(25 E)$ & 0.9900 \\
\hline$C(26 B)-H(26 D)$ & 0.9800 \\
\hline$C(26 B)-H(26 E)$ & 0.9800 \\
\hline$C(26 B)-H(26 F)$ & 0.9800 \\
\hline $\mathrm{C}(7)-\mathrm{N}(1)-\mathrm{C}(8)$ & $112.94(18)$ \\
\hline $\mathrm{C}(7)-\mathrm{N}(1)-\mathrm{C}(19)$ & $124.00(19)$ \\
\hline $\mathrm{C}(8)-\mathrm{N}(1)-\mathrm{C}(19)$ & $123.0(2)$ \\
\hline$C(12)-C(1)-C(2)$ & $121.4(2)$ \\
\hline $\mathrm{C}(12)-\mathrm{C}(1)-\mathrm{H}(1)$ & $118.2(13)$ \\
\hline $\mathrm{C}(2)-\mathrm{C}(1)-\mathrm{H}(1)$ & $120.4(13)$ \\
\hline$C(3)-C(2)-C(1)$ & $120.2(2)$ \\
\hline $\mathrm{C}(3)-\mathrm{C}(2)-\mathrm{H}(2)$ & $119.8(14)$ \\
\hline $\mathrm{C}(1)-\mathrm{C}(2)-\mathrm{H}(2)$ & $120.0(14)$ \\
\hline$C(2)-C(3)-C(4)$ & $120.8(2)$ \\
\hline $\mathrm{C}(2)-\mathrm{C}(3)-\mathrm{H}(3)$ & $119.8(14)$ \\
\hline $\mathrm{C}(4)-\mathrm{C}(3)-\mathrm{H}(3)$ & $119.2(14)$ \\
\hline$C(3)-C(4)-C(11)$ & $119.13(19)$ \\
\hline$C(3)-C(4)-C(5)$ & $120.81(19)$ \\
\hline$C(11)-C(4)-C(5)$ & $120.06(19)$ \\
\hline$C(6)-C(5)-C(4)$ & $116.96(19)$ \\
\hline$C(6)-C(5)-C(13 A)$ & $118.8(3)$ \\
\hline$C(4)-C(5)-C(13 A)$ & $123.4(3)$ \\
\hline$C(6)-C(5)-C(13 B)$ & $125.2(3)$ \\
\hline$C(4)-C(5)-C(13 B)$ & $117.6(3)$ \\
\hline$C(13 A)-C(5)-C(13 B)$ & $14.6(3)$ \\
\hline$C(5)-C(6)-C(9)$ & $123.57(19)$ \\
\hline$C(5)-C(6)-C(7)$ & $128.1(2)$ \\
\hline$C(9)-C(6)-C(7)$ & $108.34(19)$ \\
\hline $\mathrm{O}(2)-\mathrm{C}(7)-\mathrm{N}(1)$ & $124.1(2)$ \\
\hline$O(2)-C(7)-C(6)$ & $130.1(2)$ \\
\hline$N(1)-C(7)-C(6)$ & $105.82(18)$ \\
\hline $\mathrm{O}(1)-\mathrm{C}(8)-\mathrm{N}(1)$ & $122.8(2)$ \\
\hline
\end{tabular}


Supporting Information

\begin{tabular}{|c|c|}
\hline$O(1)-C(8)-C(9)$ & $130.9(2)$ \\
\hline $\mathrm{N}(1)-\mathrm{C}(8)-\mathrm{C}(9)$ & $106.26(18)$ \\
\hline$C(10)-C(9)-C(6)$ & $121.12(19)$ \\
\hline$C(10)-C(9)-C(8)$ & $132.32(19)$ \\
\hline$C(6)-C(9)-C(8)$ & $106.36(17)$ \\
\hline$C(9)-C(10)-C(11)$ & $116.58(19)$ \\
\hline$C(9)-C(10)-C(12) \# 1$ & $125.49(19)$ \\
\hline$C(11)-C(10)-C(12) \# 1$ & $117.85(18)$ \\
\hline$C(12)-C(11)-C(4)$ & $118.79(19)$ \\
\hline$C(12)-C(11)-C(10)$ & $120.21(19)$ \\
\hline$C(4)-C(11)-C(10)$ & $121.00(19)$ \\
\hline$C(1)-C(12)-C(11)$ & $118.98(19)$ \\
\hline$C(1)-C(12)-C(10) \# 1$ & $121.93(19)$ \\
\hline$C(11)-C(12)-C(10) \# 1$ & $119.09(18)$ \\
\hline$C(18 A)-C(13 A)-C(14 A)$ & $118.3(5)$ \\
\hline$C(18 A)-C(13 A)-C(5)$ & $124.9(5)$ \\
\hline$C(14 A)-C(13 A)-C(5)$ & $116.8(4)$ \\
\hline$C(15 A)-C(14 A)-C(13 A)$ & $119.7(5)$ \\
\hline$C(15 A)-C(14 A)-H(14 A)$ & 120.1 \\
\hline$C(13 A)-C(14 A)-H(14 A)$ & 120.1 \\
\hline$C(14 A)-C(15 A)-C(16 A)$ & $119.8(5)$ \\
\hline$C(14 A)-C(15 A)-H(15 A)$ & 120.1 \\
\hline$C(16 A)-C(15 A)-H(15 A)$ & 120.1 \\
\hline$C(17 A)-C(16 A)-C(15 A)$ & $119.5(5)$ \\
\hline$C(17 A)-C(16 A)-H(16 A)$ & 120.2 \\
\hline$C(15 A)-C(16 A)-H(16 A)$ & 120.2 \\
\hline$C(18 A)-C(17 A)-C(16 A)$ & $120.4(5)$ \\
\hline$C(18 A)-C(17 A)-H(17 A)$ & 119.8 \\
\hline$C(16 A)-C(17 A)-H(17 A)$ & 119.8 \\
\hline$C(17 A)-C(18 A)-C(13 A)$ & $121.9(6)$ \\
\hline $\mathrm{C}(17 \mathrm{~A})-\mathrm{C}(18 \mathrm{~A})-\mathrm{H}(18 \mathrm{~A})$ & 119.1 \\
\hline $\mathrm{C}(13 \mathrm{~A})-\mathrm{C}(18 \mathrm{~A})-\mathrm{H}(18 \mathrm{~A})$ & 119.1 \\
\hline$C(14 B)-C(13 B)-C(18 B)$ & $118.8(6)$ \\
\hline$C(14 B)-C(13 B)-C(5)$ & $129.2(5)$ \\
\hline$C(18 B)-C(13 B)-C(5)$ & $111.9(6)$ \\
\hline$C(13 B)-C(14 B)-C(15 B)$ & $121.4(6)$ \\
\hline$C(13 B)-C(14 B)-H(14 B)$ & 119.3 \\
\hline$C(15 B)-C(14 B)-H(14 B)$ & 119.3 \\
\hline$C(16 B)-C(15 B)-C(14 B)$ & $121.9(7)$ \\
\hline$C(16 B)-C(15 B)-H(15 B)$ & 119.0 \\
\hline$C(14 B)-C(15 B)-H(15 B)$ & 119.0 \\
\hline$C(15 B)-C(16 B)-C(17 B)$ & $121.2(7)$ \\
\hline$C(15 B)-C(16 B)-H(16 B)$ & 119.4 \\
\hline$C(17 B)-C(16 B)-H(16 B)$ & 119.4 \\
\hline$C(16 B)-C(17 B)-C(18 B)$ & $118.6(6)$ \\
\hline $\mathrm{C}(16 \mathrm{~B})-\mathrm{C}(17 \mathrm{~B})-\mathrm{H}(17 \mathrm{~B})$ & 120.7 \\
\hline$C(18 B)-C(17 B)-H(17 B)$ & 120.7 \\
\hline$C(13 B)-C(18 B)-C(17 B)$ & $117.4(7)$ \\
\hline $\mathrm{C}(13 \mathrm{~B})-\mathrm{C}(18 \mathrm{~B})-\mathrm{H}(18 \mathrm{~B})$ & 121.3 \\
\hline $\mathrm{C}(17 \mathrm{~B})-\mathrm{C}(18 \mathrm{~B})-\mathrm{H}(18 \mathrm{~B})$ & 121.3 \\
\hline $\mathrm{N}(1)-\mathrm{C}(19)-\mathrm{C}(20)$ & $112.8(2)$ \\
\hline $\mathrm{N}(1)-\mathrm{C}(19)-\mathrm{H}(19 \mathrm{~B})$ & $105.5(15)$ \\
\hline $\mathrm{C}(20)-\mathrm{C}(19)-\mathrm{H}(19 \mathrm{~B})$ & $107.1(15)$ \\
\hline $\mathrm{N}(1)-\mathrm{C}(19)-\mathrm{H}(19 \mathrm{~A})$ & $106.4(15)$ \\
\hline$C(20)-C(19)-H(19 A)$ & $110.5(15)$ \\
\hline $\mathrm{H}(19 \mathrm{~B})-\mathrm{C}(19)-\mathrm{H}(19 \mathrm{~A})$ & $115(2)$ \\
\hline$C(19)-C(20)-C(21)$ & $111.2(2)$ \\
\hline
\end{tabular}


Supporting Information

\begin{tabular}{|c|c|}
\hline $\mathrm{C}(19)-\mathrm{C}(20)-\mathrm{H}(20 \mathrm{~B})$ & $107.7(15)$ \\
\hline $\mathrm{C}(21)-\mathrm{C}(20)-\mathrm{H}(20 \mathrm{~B})$ & $107.7(16)$ \\
\hline$C(19)-C(20)-H(20 A)$ & $108.3(15)$ \\
\hline $\mathrm{C}(21)-\mathrm{C}(20)-\mathrm{H}(20 \mathrm{~A})$ & $109.4(15)$ \\
\hline $\mathrm{H}(20 \mathrm{~B})-\mathrm{C}(20)-\mathrm{H}(20 \mathrm{~A})$ & $113(2)$ \\
\hline$C(22)-C(21)-C(20)$ & $114.9(2)$ \\
\hline $\mathrm{C}(22)-\mathrm{C}(21)-\mathrm{H}(21 \mathrm{~B})$ & $109.4(18)$ \\
\hline $\mathrm{C}(20)-\mathrm{C}(21)-\mathrm{H}(21 \mathrm{~B})$ & $108.2(18)$ \\
\hline$C(22)-C(21)-H(21 A)$ & $110.7(19)$ \\
\hline $\mathrm{C}(20)-\mathrm{C}(21)-\mathrm{H}(21 \mathrm{~A})$ & $104.3(19)$ \\
\hline $\mathrm{H}(21 \mathrm{~B})-\mathrm{C}(21)-\mathrm{H}(21 \mathrm{~A})$ & $109(3)$ \\
\hline$C(21)-C(22)-C(23)$ & $113.5(2)$ \\
\hline $\mathrm{C}(21)-\mathrm{C}(22)-\mathrm{H}(22 \mathrm{~A})$ & 108.9 \\
\hline $\mathrm{C}(23)-\mathrm{C}(22)-\mathrm{H}(22 \mathrm{~A})$ & 108.9 \\
\hline$C(21)-C(22)-H(22 B)$ & 108.9 \\
\hline $\mathrm{C}(23)-\mathrm{C}(22)-\mathrm{H}(22 \mathrm{~B})$ & 108.9 \\
\hline $\mathrm{H}(22 \mathrm{~A})-\mathrm{C}(22)-\mathrm{H}(22 \mathrm{~B})$ & 107.7 \\
\hline$C(24 B)-C(23)-C(22)$ & $115.9(3)$ \\
\hline$C(24 B)-C(23)-C(24 A)$ & $29.5(3)$ \\
\hline$C(22)-C(23)-C(24 A)$ & $113.4(3)$ \\
\hline$C(24 B)-C(23)-H(23 B)$ & $121(2)$ \\
\hline$C(22)-C(23)-H(23 B)$ & $108(2)$ \\
\hline$C(24 A)-C(23)-H(23 B)$ & $98(2)$ \\
\hline$C(24 B)-C(23)-H(23 A)$ & $93(2)$ \\
\hline$C(22)-C(23)-H(23 A)$ & $104.8(19)$ \\
\hline$C(24 A)-C(23)-H(23 A)$ & $120(2)$ \\
\hline $\mathrm{H}(23 \mathrm{~B})-\mathrm{C}(23)-\mathrm{H}(23 \mathrm{~A})$ & $112(3)$ \\
\hline$C(25 A)-C(24 A)-C(23)$ & $114.4(5)$ \\
\hline$C(25 A)-C(24 A)-H(24 A)$ & 108.7 \\
\hline $\mathrm{C}(23)-\mathrm{C}(24 \mathrm{~A})-\mathrm{H}(24 \mathrm{~A})$ & 108.7 \\
\hline$C(25 A)-C(24 A)-H(24 B)$ & 108.7 \\
\hline $\mathrm{C}(23)-\mathrm{C}(24 \mathrm{~A})-\mathrm{H}(24 \mathrm{~B})$ & 108.7 \\
\hline $\mathrm{H}(24 \mathrm{~A})-\mathrm{C}(24 \mathrm{~A})-\mathrm{H}(24 \mathrm{~B})$ & 107.6 \\
\hline$C(24 A)-C(25 A)-C(26 A)$ & $109.2(6)$ \\
\hline$C(24 A)-C(25 A)-H(25 A)$ & 109.8 \\
\hline$C(26 A)-C(25 A)-H(25 A)$ & 109.8 \\
\hline$C(24 A)-C(25 A)-H(25 B)$ & 109.8 \\
\hline$C(26 A)-C(25 A)-H(25 B)$ & 109.8 \\
\hline $\mathrm{H}(25 \mathrm{~A})-\mathrm{C}(25 \mathrm{~A})-\mathrm{H}(25 \mathrm{~B})$ & 108.3 \\
\hline$C(25 A)-C(26 A)-H(26 A)$ & 109.5 \\
\hline$C(25 A)-C(26 A)-H(26 B)$ & 109.5 \\
\hline$H(26 A)-C(26 A)-H(26 B)$ & 109.5 \\
\hline$C(25 A)-C(26 A)-H(26 C)$ & 109.5 \\
\hline$H(26 A)-C(26 A)-H(26 C)$ & 109.5 \\
\hline$H(26 B)-C(26 A)-H(26 C)$ & 109.5 \\
\hline$C(23)-C(24 B)-C(25 B)$ & $113.1(5)$ \\
\hline $\mathrm{C}(23)-\mathrm{C}(24 \mathrm{~B})-\mathrm{H}(24 \mathrm{C})$ & 109.0 \\
\hline$C(25 B)-C(24 B)-H(24 C)$ & 109.0 \\
\hline$C(23)-C(24 B)-H(24 D)$ & 109.0 \\
\hline$C(25 B)-C(24 B)-H(24 D)$ & 109.0 \\
\hline $\mathrm{H}(24 \mathrm{C})-\mathrm{C}(24 \mathrm{~B})-\mathrm{H}(24 \mathrm{D})$ & 107.8 \\
\hline$C(26 B)-C(25 B)-C(24 B)$ & $113.2(6)$ \\
\hline$C(26 B)-C(25 B)-H(25 D)$ & 108.9 \\
\hline$C(24 B)-C(25 B)-H(25 D)$ & 108.9 \\
\hline$C(26 B)-C(25 B)-H(25 E)$ & 108.9 \\
\hline$C(24 B)-C(25 B)-H(25 E)$ & 108.9 \\
\hline $\mathrm{H}(25 \mathrm{D})-\mathrm{C}(25 \mathrm{~B})-\mathrm{H}(25 \mathrm{E})$ & 107.8 \\
\hline
\end{tabular}




$$
\begin{array}{ll}
\mathrm{C}(25 B)-\mathrm{C}(26 \mathrm{~B})-\mathrm{H}(26 \mathrm{D}) & 109.5 \\
\mathrm{C}(25 B)-\mathrm{C}(26 \mathrm{~B})-\mathrm{H}(26 \mathrm{E}) & 109.5 \\
\mathrm{H}(26 \mathrm{D})-\mathrm{C}(26 \mathrm{~B})-\mathrm{H}(26 \mathrm{E}) & 109.5 \\
\mathrm{C}(25 B)-\mathrm{C}(26 \mathrm{~B})-\mathrm{H}(26 \mathrm{~F}) & 109.5 \\
\mathrm{H}(26 \mathrm{D})-\mathrm{C}(26 \mathrm{~B})-\mathrm{H}(26 \mathrm{~F}) & 109.5 \\
\mathrm{H}(26 \mathrm{E})-\mathrm{C}(26 \mathrm{~B})-\mathrm{H}(26 \mathrm{~F}) & 109.5
\end{array}
$$

\begin{tabular}{|c|c|c|c|c|c|c|}
\hline & U11 & U22 & U33 & U23 & U13 & U12 \\
\hline O (1) & $39(1)$ & $56(1)$ & $45(1)$ & $-7(1)$ & $2(1)$ & $11(1)$ \\
\hline$O(2)$ & 31 (1) & $94(2)$ & $47(1)$ & $24(1)$ & $-6(1)$ & $4(1)$ \\
\hline $\mathrm{N}(1)$ & 27 (1) & $40(1)$ & $45(1)$ & $6(1)$ & $0(1)$ & $4(1)$ \\
\hline C (1) & $28(1)$ & $39(1)$ & $33(1)$ & $3(1)$ & $1(1)$ & $-2(1)$ \\
\hline C (2) & $35(1)$ & $49(1)$ & $29(1)$ & $1(1)$ & $3(1)$ & $-1(1)$ \\
\hline C (3) & 37 (1) & $51(1)$ & $25(1)$ & $6(1)$ & $-2(1)$ & $-5(1)$ \\
\hline C (4) & $30(1)$ & 37 (1) & 31 (1) & $8(1)$ & $-1(1)$ & $-4(1)$ \\
\hline C (5) & 31 (1) & $41(1)$ & $34(1)$ & $11(1)$ & $-2(1)$ & $-6(1)$ \\
\hline$C(6)$ & $28(1)$ & $38(1)$ & 37 (1) & $10(1)$ & $-2(1)$ & $-3(1)$ \\
\hline C (7) & 31 (1) & $45(1)$ & $42(1)$ & $14(1)$ & $-3(1)$ & $-1(1)$ \\
\hline C ( 8$)$ & 31 (1) & $32(1)$ & $41(1)$ & $3(1)$ & $1(1)$ & $2(1)$ \\
\hline$C(9)$ & $27(1)$ & 31 (1) & $35(1)$ & $5(1)$ & $0(1)$ & $-1(1)$ \\
\hline$C(10)$ & $29(1)$ & $25(1)$ & $32(1)$ & $1(1)$ & $-2(1)$ & $-2(1)$ \\
\hline C (11) & $28(1)$ & $28(1)$ & $32(1)$ & $5(1)$ & $1(1)$ & $-3(1)$ \\
\hline C (12) & $29(1)$ & $30(1)$ & $29(1)$ & $3(1)$ & $-1(1)$ & $-1(1)$ \\
\hline C (19) & $29(1)$ & $41(1)$ & $57(2)$ & $6(1)$ & $3(1)$ & $6(1)$ \\
\hline$C(20)$ & $36(1)$ & $40(1)$ & $52(2)$ & $2(1)$ & $5(1)$ & $6(1)$ \\
\hline$C(21)$ & $38(1)$ & $41(1)$ & $62(2)$ & $0(1)$ & $10(1)$ & $4(1)$ \\
\hline C (22) & $52(2)$ & $47(2)$ & $95(2)$ & $7(2)$ & $30(2)$ & $10(1)$ \\
\hline C (23) & $53(2)$ & $51(2)$ & $89(2)$ & $-2(2)$ & $27(2)$ & $4(1)$ \\
\hline
\end{tabular}

Symmetry transformations used to generate equivalent atoms: $\# 1-\mathrm{x}+2, \mathrm{y},-\mathrm{z}+1 / 2$

Table 4. Anisotropic displacement parameters $\left(\mathrm{A}^{2} \times 10^{3}\right)$ for 1. Anisotropic displacement factor exponent takes the form: $-2 \mathrm{pi}^{2}\left[\mathrm{~h}^{2} \mathrm{a}^{*^{2}} \mathrm{U} 11+\ldots+2 \mathrm{~h} \mathrm{k} \mathrm{a}^{*} \mathrm{~b}^{*} \mathrm{U} 12\right]$. 
Table 5. Hydrogen coordinates $\left(\times 10^{4}\right)$ and isotropic displacement parameters $\left(A^{2} \times 10^{3}\right)$ for 1 .

\begin{tabular}{|c|c|c|c|c|}
\hline & $x$ & y & z & $\mathrm{U}(\mathrm{eq})$ \\
\hline $\mathrm{H}(14 \mathrm{~A})$ & 8195 & 3160 & 569 & 57 \\
\hline $\mathrm{H}(15 \mathrm{~A})$ & 7751 & 3344 & -403 & 63 \\
\hline $\mathrm{H}(16 \mathrm{~A})$ & 7798 & 5791 & -931 & 30 \\
\hline $\mathrm{H}(17 \mathrm{~A})$ & 8168 & 8112 & -432 & 37 \\
\hline $\mathrm{H}(18 \mathrm{~A})$ & 8635 & 7916 & 502 & 45 \\
\hline $\mathrm{H}(14 \mathrm{~B})$ & 8522 & 2762 & 449 & 56 \\
\hline $\mathrm{H}(15 \mathrm{~B})$ & 8066 & 2804 & -505 & 72 \\
\hline $\mathrm{H}(16 \mathrm{~B})$ & 7737 & 5043 & -920 & 64 \\
\hline $\mathrm{H}(17 \mathrm{~B})$ & 8013 & 7486 & -512 & 60 \\
\hline $\mathrm{H}(18 \mathrm{~B})$ & 8500 & 7540 & 483 & 60 \\
\hline $\mathrm{H}(22 \mathrm{~A})$ & 5982 & 4087 & 3497 & 77 \\
\hline $\mathrm{H}(22 \mathrm{~B})$ & 5617 & 4079 & 2859 & 77 \\
\hline $\mathrm{H}(24 \mathrm{~A})$ & 4527 & 3738 & 3158 & 62 \\
\hline $\mathrm{H}(24 \mathrm{~B})$ & 4860 & 3151 & 3772 & 62 \\
\hline $\mathrm{H}(25 \mathrm{~A})$ & 3802 & 3896 & 3831 & 72 \\
\hline $\mathrm{H}(25 \mathrm{~B})$ & 4011 & 5732 & 3746 & 72 \\
\hline $\mathrm{H}(26 \mathrm{~A})$ & 3990 & 5155 & 4789 & 145 \\
\hline $\mathrm{H}(26 \mathrm{~B})$ & 4677 & 5509 & 4629 & 145 \\
\hline $\mathrm{H}(26 \mathrm{C})$ & 4454 & 3692 & 4718 & 145 \\
\hline $\mathrm{H}(24 \mathrm{C})$ & 5149 & 3855 & 4189 & 72 \\
\hline $\mathrm{H}(24 \mathrm{D})$ & 4733 & 3318 & 3615 & 72 \\
\hline $\mathrm{H}(25 \mathrm{D})$ & 4429 & 5879 & 4424 & 86 \\
\hline $\mathrm{H}(25 \mathrm{E})$ & 4025 & 5479 & 3828 & 86 \\
\hline $\mathrm{H}(26 \mathrm{D})$ & 3588 & 4341 & 4642 & 126 \\
\hline $\mathrm{H}(26 \mathrm{E})$ & 4201 & 3364 & 4811 & 126 \\
\hline $\mathrm{H}(26 \mathrm{~F})$ & 3805 & 2938 & 4211 & 126 \\
\hline $\mathrm{H}(1)$ & $11118(11)$ & $4360(30)$ & $1563(9)$ & $31(6)$ \\
\hline $\mathrm{H}(3)$ & $9615(11)$ & $4580(30)$ & $445(11)$ & $41(6)$ \\
\hline $\mathrm{H}(20 \mathrm{~B})$ & $6643(12)$ & $4820(30)$ & $2378(12)$ & $55(8)$ \\
\hline $\mathrm{H}(2)$ & $10643(11)$ & $4070(30)$ & $593(11)$ & $44(6)$ \\
\hline $\mathrm{H}(19 \mathrm{~B})$ & $7248(11)$ & $7500(30)$ & $2912(12)$ & $50(7)$ \\
\hline $\mathrm{H}(19 \mathrm{~A})$ & $6934(12)$ & $7480(30)$ & $2225(12)$ & $53(7)$ \\
\hline $\mathrm{H}(21 \mathrm{~B})$ & $5950(14)$ & $6690(40)$ & $2685(13)$ & $70(9)$ \\
\hline $\mathrm{H}(20 \mathrm{~A})$ & $6989(12)$ & $4840(30)$ & $3062(12)$ & $55(7)$ \\
\hline $\mathrm{H}(21 \mathrm{~A})$ & $6289(14)$ & $6770(40)$ & 3305 (14) & $73(10)$ \\
\hline $\mathrm{H}(23 \mathrm{~B})$ & $4953(17)$ & $6140(50)$ & $3185(16)$ & $97(12)$ \\
\hline $\mathrm{H}(23 \mathrm{~A})$ & $5348(15)$ & $6030(40)$ & $3844(15)$ & $77(10)$ \\
\hline
\end{tabular}




\section{References}

(1) Kamahori, K.; Tada, S.; Ito, K.; Itsuno, S. Macromolecules 1999, 32, 541.

(2) Demas, J. N.; Crosby, G. A. J. Phys.Chem.1971, 75, 991.

(3) Damrauer, N. H.; Boussie, T. R.; Devenney, M.; McCusker, J. K. J. Am. Chem. Soc. 1997, $119,8253$.

(4) (a) Debad, J. D.; Morris, J. C.; Lynch, V.; Magnus, P.; Bard, A. J. J. Am. Chem. Soc. 1996, 118, 2374. (b)Debad, J. D.; Morris, J. C..; Magnus, P.; Bard, A. J. J. Org. Chem. 1997, 62, 530. 